\title{
FIXED POINTS OF ALMOST GENERALIZED $(\alpha, \beta)-(\psi, \varphi)$-CONTRACTIVE MAPPINGS IN $b$-METRIC SPACES
}

\author{
Gutti Venkata Ravindranadh Babu ${ }^{1}$ and Tolera Mosissa Dula ${ }^{2}$
}

\begin{abstract}
In this paper, we introduce almost generalized $(\alpha, \beta)-(\psi, \varphi)$-contractive maps, and prove some new fixed point results for this class of mappings in $b$-metric spaces. We provide examples in support of our results. Our results extend/generalize the results of Dutta and Choudhury [8] and Yamaod and Sintunavarat [14].
\end{abstract}

Keywords: $b$-metric space, cyclic $(\alpha, \beta)$-admissible mapping, almost generalized $(\alpha, \beta)$ $(\psi, \varphi)$-contractive mappings, fixed point.

\section{Introduction}

The development of fixed point theory is based on the generalization of contraction conditions in one direction or/and generalization of metric space. Banach contraction principle is one of the most useful results in fixed point theory. In the direction of generalization of contraction conditions, in 1997, Alber and

Guerre-Delabriere [1] introduced weakly contractive maps, which are extensions of contraction maps, and obtained fixed point results in the setting of Hilbert spaces. Rhoades [12] extended this concept to metric spaces. In 2008, Dutta and

Choudhury [8] introduced $(\psi, \varphi)$ - weakly contractive maps and proved the existence of fixed points in complete metric spaces. In continuation to the extensions of contraction maps, Berinde [4] initiated the concept, namely 'weak contractions', which are renamed 'almost contractions', and established fixed point results. For more work on almost contractions, we refer the reader to [3], [5], [8] and [12].

On the other hand, in the direction of generalization of metric spaces, in 1993, Czerwik [7] introduced the concept of $b$-metric spaces and proved the Banach contraction mapping principle in this setting, where $b$-metric need not be continuous. Afterwards, many mathematicians studied fixed point theorems for single-valued and multi-valued mappings in b-metric spaces. In 2014, Alizadeh, Moradlou and

Received April 20, 2017; accepted September 21, 2017

2010 Mathematics Subject Classification. 
Peyman [2] introduced the notation of cyclic $(\alpha, \beta)$-admissible mappings and proved some fixed point results in the setting of complete metric spaces.

The paper is organized as follows. In Section 2, we present preliminaries and earlier papers that we require to develop the main results. In fact, motivated by the work by Alizadeh, Moradlou and Peyman [2], Berinde [4] and Dutta and Choudhury [8], we introduce almost generalized $(\alpha, \beta)-(\psi, \varphi)$-contractive mappings in this section. In Section 3, we prove our main results in which we study the existence of fixed points of almost generalized $(\alpha, \beta)-(\psi, \varphi)$-contractive mappings. In Section 4, we provide examples in support of our results. Our results extend/generalize the results of Dutta and Choudhury [8] and Yamaod and Sintunavarat [14].

\section{Preliminaries}

Throughout this paper, $\mathbf{R}$ denotes the real line, and $\mathbf{N}$ is the set of all natural numbers.

In this section, we mention some well-known notations, definitions and known results in the literature that we use in the sequel.

Definition 2.1. [10] A function $\psi:[0, \infty) \rightarrow[0, \infty)$ is called an altering distance function if the following properties hold:

(i) $\psi$ is a continuous and nondecreasing function, and

(ii) $\psi(t)=0$ if and only if $t=0$.

We denote the class of all altering distance functions by $\Psi$

Definition 2.2. [7] Let $\mathrm{X}$ be a non-empty set. A function $d: X \times X \rightarrow[0, \infty)$ is said to be a $b$-metric if the following conditions are satisfied;

(i) $0 \leq d(x, y)$ for all $x, y \in X$ and $d(x, y)=0$ if and only if $x=y$,

(ii) $d(x, y)=d(y, x)$ for all $x, y \in X$,

(iii) there exists $s \geq 1$ such that $d(x, z) \leq s[d(x, y)+d(y, z)]$ for all $x, y, z \in X$.

In this case, the pair $(X, d)$ is called a $b$-metric space with the coefficient $s$.

Every metric space is a $b$-metric space with $s=1$. In general, every $b$-metric space is not a metric space.

Example 2.1. Let $X=\mathbf{R}$, and let the mapping $d: X \times X \rightarrow[0, \infty)$ be defined by $d(x, y)=|x-y|^{2}$ for all $x, y \in X$. Then $(X, d)$ is a $b$-metric space with coefficient $s=2$, but it is not a metric.

Example 2.2. Let $0<p<1$. We write $l_{p}(\mathbf{R})=\left\{\left.\left\{x_{n}\right\} \subseteq \mathbf{R}\left|\sum_{n=1}^{\infty}\right| x_{n}\right|^{p}<\infty\right\}$, and define $d: l_{p}(\mathbf{R}) \times l_{p}(\mathbf{R}) \rightarrow[0, \infty)$ by $d(x, y)=\left(\sum_{n=1}^{\infty}\left|x_{n}-y_{n}\right|^{p}\right)^{\frac{1}{p}}$ for $x=\left\{x_{n}\right\}, y=\left\{y_{n}\right\}$ in $l_{p}(\mathbf{R})$. Then this $d$ is a $b$-metric with the coefficient $s=2^{\frac{1}{p}}>1$.

Remark 2.1. A $b$-metric need not be a continuous function. For more details, we refer [9]. 
Definition 2.3. $[6]$ Let $(X, d)$ be a $b$-metric space.

(i) A sequence $\left\{x_{n}\right\}$ in $\mathrm{X}$ is called $b$-convergent if there exists $x \in X$ such that $d\left(x_{n}, x\right) \rightarrow 0$ as $n \rightarrow \infty$. In this case, we write $\lim _{n \rightarrow \infty} x_{n}=x$.

(ii) A sequence $\left\{x_{n}\right\}$ in $X$ is called $b$-Cauchy if $d\left(x_{n}, x_{m}\right) \rightarrow 0$ as $n, m \rightarrow \infty$.

(iii) The $b$-metric space $(X, d)$ is said to be $b$-complete if every $b$-Cauchy sequence in $\mathrm{X}$ is $b$-convergent. In this case, we say that $(X, d)$ is a complete $b$-metric space. That is, a $b$-metric space which is $b$-complete is a complete $b$-metric space.

Lemma 2.1. [9] Let $(X, d)$ be a $b$-metric space with $s \geq 1$.

(i) If a sequence $\left\{x_{n}\right\} \subset X$ is a $b$-convergent sequence, then it admits a unique limit.

(ii) Every $b$-convergent sequence in $X$ is $b$-Cauchy.

Definition 2.4. [6] Let $(X, d)$ and $\left(Y, d^{\prime}\right)$ be two $b$-metric spaces. A function $f: X \rightarrow Y$ is $b$-continuous at $x \in X$ if it is $b$-sequentially continuous at $X$. That is, whenever $\left\{x_{n}\right\}$ is $b$-convergent to $x,\left\{f x_{n}\right\}$ is $b$-convergent to $f x$.

Definition 2.5. [11] Let $A$ and $B$ be nonempty subsets of $X$. A mapping $f: A \cup B \rightarrow A \cup B$ is said to be cyclic if $f(A) \subset B$ and $f(B) \subset A$.

In the context of the metric space setting, weakly contractive maps are weaker than the contraction maps [[1], [12]].

Theorem 2.1. [8] Let $(X, d)$ be a complete metric space and $f: X \rightarrow X$ be a selfmap of $X$. If there exist $\psi, \varphi$ in $\Psi$ such that

$$
\psi(d(f x, f y)) \leq \psi(d(x, y))-\varphi(d(x, y)) \text { for all } x, y \in X .
$$

then $f$ has a unique fixed point.

Here we note that if $\psi(t)=t \geq 0$ in (2.1) then we say that $f$ is a weakly contractive map on $X$, and hence weakly contractive maps are a special case of the maps satisfying the inequality $(2.1)$.

Definition 2.6. [2] Let $X$ be a nonempty set, $f$ be a selfmap on $X$ and $\alpha, \beta: X \rightarrow[0, \infty)$ be two mappings. We say that $f$ is a cyclic $(\alpha, \beta)$-admissible mapping if

(i) for any $x \in X$ with $\alpha(x) \geq 1 \Longrightarrow \beta(f x) \geq 1$, and

(ii) for any $y \in X$ with $\beta(y) \geq 1 \Longrightarrow \alpha(f y) \geq 1$.

In the metric space setting, Alizadeh, Moradlou and Peyman [2] defined $(\alpha, \beta)-(\psi, \varphi)$-contractive mappings as follows.

Definition 2.7. [2] Let $(X, d)$ be a metric space and $f: X \rightarrow X$ be a cyclic $(\alpha, \beta)$ - admissible mapping. We say that $f: X \rightarrow X$ is an $(\alpha, \beta)-(\psi, \varphi)$ - contractive mapping if

$$
\begin{aligned}
x, y & \in X \text { with } \alpha(x) \beta(y) \geq 1 \\
& \Longrightarrow \psi(d(f x, f y)) \leq \psi(d(x, y))-\varphi(d(x, y))
\end{aligned}
$$


where

$\psi:[0, \infty) \rightarrow[0, \infty)$ is a continuous and increasing function and

$\varphi:[0, \infty) \rightarrow[0, \infty)$ is a lower semi-continuous function such that $\varphi(t)=0$

if and only if $t=0$.

Theorem 2.2. $[2]$ Let $(X, d)$ be a complete metric space, $\alpha, \beta: X \rightarrow[0, \infty)$ be two mappings and $f: X \rightarrow X$ be an $(\alpha, \beta)-(\psi, \varphi)$-contractive mapping. Suppose that the following conditions hold:

(i) there exists $x_{0} \in X$ such that $\alpha\left(x_{0}\right) \geq 1$ and $\beta\left(x_{0}\right) \geq 1$. If either

(ii) $f$ is continuous or

(iii) if $\left\{x_{n}\right\}$ is a sequence in $X$ such that $x_{n} \rightarrow x$ and $\beta\left(x_{n}\right) \geq 1$ for all $n$, then $\beta(x) \geq 1$

then $f$ has a fixed point in $X$.

Moreover, if $\alpha(x) \geq 1$ and $\beta(x) \geq 1$ for all $x, y \in \operatorname{Fix}(f)$, where $\operatorname{Fix}(f)$ is the set of all fixed points of $f$, then $f$ has a unique fixed point.

Very recently, Yamaod and Sintunavarat [14] introduced $(\alpha, \beta)-(\psi, \varphi)$-contractive mappings in $b$-metric spaces as follows:

Definition 2.8. [14] Let $(X, d)$ be a b-metric space with the coefficient $s \geq 1$ and let $\alpha, \beta: X \rightarrow[0, \infty)$ be two given mappings. We say that $f: X \rightarrow \bar{X}$ is an $(\alpha, \beta)-(\psi, \varphi)$ - contractive mapping if the following condition holds:

for any $x, y \in X$ with $\alpha(x) \beta(y) \geq 1$ implies

$$
\psi\left(s^{3} d(f x, f y)\right) \leq \psi\left(M_{s}(x, y)\right)-\varphi\left(M_{s}(x, y)\right)
$$

where

$$
\begin{aligned}
& M_{s}(x, y)=\max \left\{d(x, y), d(x, f x), d(y, f y), \frac{d(x, f y)+d(y, f x)}{2 s}\right\} \text { and } \\
& \psi, \varphi:[0, \infty) \rightarrow[0, \infty) \text { are altering distance functions. }
\end{aligned}
$$

Theorem 2.3. [14] Let $(X, d)$ be a complete $b$-metric space with the coefficient $s \geq 1, \alpha, \beta: X \rightarrow[0, \infty)$ be two mappings and $f: X \rightarrow X$ be an $(\alpha, \beta)-(\psi, \varphi)$-contractive mapping. Suppose that

(1) one of the following condition holds;

(1.1) there exists $x_{0} \in X$ such that $\alpha\left(x_{0}\right) \geq 1$

(1.2) There exists $y_{0} \in X$ such that $\beta\left(y_{0}\right) \geq 1$

(2) $f$ is continuous

(3) $f$ is cyclic $(\alpha, \beta)$-admissible mapping.

Then $f$ has a fixed point. Moreover, if the sequence $\left\{x_{n}\right\}$ in $\mathrm{X}$ defined by $x_{n}=f x_{n-1}$ for all $n \in \mathbb{N}$ is such that $x_{0}$ is an initial point in the condition (1.1) and the sequence $\left\{y_{n}\right\}$ in $\mathrm{X}$ defined by $y_{n}=f y_{n-1}$ for all $n \in \mathbb{N}$ is such that $y_{0}$ is an initial point in the condition (1.2) then $\left\{x_{n}\right\}$ and $\left\{y_{n}\right\}$ converge to a fixed point of $f$.

Remark 2.2. While proving the Cauchy part of Theorem 2.3, the authors Yamaoda and Sintunavarat [14] claimed the following: 
"If $\left\{x_{n}\right\}$ is not Cauchy, then there exists an $\epsilon>0$ for which we can find subsequences $\left\{x_{m(k)}\right\}$ and $\left\{x_{n(k)}\right\}$ such that $n(k)>m(k) \geq k, m(k)$ is even and $n(k)$ is odd, $d\left(x_{m(k)}, x_{n(k)}\right) \geq \epsilon$ and $n(k)$ is the smallest number such that $d\left(x_{m(k)}, x_{n(k)}\right) \geq \epsilon$ and $d\left(x_{m(k)}, x_{n(k)-1}\right)<\epsilon . "$

But ' $m(k)$ is even and $n(k)$ is odd' may not be possible due to the following example:

Example 2.3. Let $X=\mathbb{R}$ with the $b$-metric defined by $d(x, y)=|x-y|^{2}, x, y \in \mathbb{R}$. We define the sequence $\left\{x_{n}\right\}$ in $X$ by

$x_{n}= \begin{cases}3^{n} & \text { if } n=1,3,5,7, \ldots \\ 3^{n+1} & \text { if } n=2,4,6,8, \ldots\end{cases}$

Then clearly the sequence $\left\{x_{n}\right\}$ is not b-Cauchy. Let $\epsilon>0$. If $\{m(k)\}$ and $\{n(k)\}$ are sequences with $m(k)$ is even and $n(k)$ is odd with $n(k)>m(k) \geq k$ and $n(k)$ is the smallest number such that $d\left(x_{m(k)}, x_{n(k)}\right) \geq \epsilon$, then we have

$n(k) \neq m(k)+1$, since $d\left(x_{m(k)}, x_{n(k)}\right)=d\left(x_{m(k)}, x_{m(k)+1}\right)=0$.

Now, $d\left(x_{m(k)}, x_{n(k)}\right)=d\left(3^{m(k)+1}, 3^{n(k)}\right) \geq \epsilon$. But

$d\left(x_{m(k)}, x_{n(k)-1}\right)=d\left(3^{m(k)+1}, 3^{n(k)-1+1}\right)=d\left(3^{m(k)+1}, 3^{n(k)}\right) \nless \epsilon$. Hence in the negation of the Cauchy part, it is not possible to mention that " $m(k)$ is even and $n(k)$ is odd ".

Thus, in order to get the valid argument, to prove the Cauchy part of the sequence $\left\{x_{n}\right\}$ of Theorem 2.3, we replace the condition (1) of Theorem 2.3 by the following:

(H): " there exists $x_{0}$ in $X$ such that $\alpha\left(x_{0}\right) \geq 1$ and $\beta\left(x_{0}\right) \geq 1 "$.

Thus the modified version of Theorem 2.3 is the following, and since it follows as a corollary to Theorem 3.1 (we prove Theorem 3.1 in Section 3) and the proof of the Cauchy part of Theorem 3.1 is proved without using the property ' $m(k)$ is even and $n(k)$ is odd' (Remark 2.2), we just state this result without proof.

Theorem 2.4. Let $(X, d)$ be a complete $b$-metric space with the coefficient $s \geq 1, \alpha, \beta: X \rightarrow[0, \infty)$ be two mappings and $f: X \rightarrow X$ be an

$(\alpha, \beta)-(\psi, \varphi)$-contractive mapping. Suppose that

(1) there exists $x_{0} \in X$ such that $\alpha\left(x_{0}\right) \geq 1$ and $\beta\left(x_{0}\right) \geq 1$

(2) $f$ is continuous

(3) $f$ is cyclic $(\alpha, \beta)$-admissible mapping.

Then $f$ has a fixed point.

Moreover, for $x_{0} \in X$ which is as in (1), if the sequence $\left\{x_{n}\right\}$ in $\mathrm{X}$ defined by $x_{n+1}=f x_{n}$ then the sequence $\left\{x_{n}\right\}$ is Cauchy and $\left\{x_{n}\right\}$ converges to a fixed point of $f$.

Now, we introduce almost generalized $(\alpha, \beta)-(\psi, \varphi)$-contractive mappings in $b$-metric spaces in the following:

Definition 2.9. Let $(X, d)$ be a $b$-metric space with the coefficient $s \geq 1$, and let $\alpha, \beta: X \rightarrow[0, \infty)$ be two given mappings. Let $f: X \rightarrow X$ be a selfmap of $X$. If 
there exist $\psi, \varphi \in \Psi$ and $L \geq 0$ such that

$$
\begin{aligned}
& \text { for all } x, y \in X \text { with } \alpha(x) \beta(y) \geq 1 \\
& \quad \Longrightarrow \psi\left(s^{3} d(f x, f y)\right) \leq \psi\left(M_{s}(x, y)\right)-\varphi\left(M^{\prime}(x, y)\right)+L N(x, y),
\end{aligned}
$$

where

$$
\begin{aligned}
M_{s}(x, y) & =\max \left\{d(x, y), d(x, f x), d(y, f y), \frac{d(x, f y)+d(y, f x)}{2 s}\right\} \\
M^{\prime}(x, y) & =\max \{d(x, y), d(y, f y)\} \text { and } \\
N(x, y) & =\min \{d(x, f x), d(y, f x)\}
\end{aligned}
$$

then we say that $f$ is an almost generalized $(\alpha, \beta)-(\psi, \varphi)$ - contractive mapping.

Here we note that if $L=0$ in (2.4), it becomes:

$$
\begin{aligned}
& \text { for all } x, y \in X \text { with } \alpha(x) \beta(y) \geq 1 \\
& \qquad \Longrightarrow \psi\left(s^{3} d(f x, f y)\right) \leq \psi\left(M_{s}(x, y)\right)-\varphi\left(M^{\prime}(x, y)\right) .
\end{aligned}
$$

Further, by the nondecreasing nature of $\varphi$, the inequality (2.3) implies (2.5) so that the inequality (2.5) is weaker than (2.3).

Example 2.4. Let $X=[0,1] \cup\{2,3, \ldots$,$\} . We define d: X \times X \rightarrow[0, \infty)$ by

$$
d(n, m)=\left\{\begin{array}{lll}
0 & \text { if } & n=m \\
\left|\frac{1}{n}-\frac{1}{m}\right| & \text { if } & n, m \in\{2,4,6, \ldots\} \\
5 & \text { if } & n, m \in\{1,3,5, \ldots\} \\
2 & \text { otherwise }
\end{array}\right.
$$

Clearly $d$ is a $b$-metric space with the coefficient $s=\frac{5}{4}$. Now, we define $f: X \rightarrow X$ by

$$
f(x)=\left\{\begin{array}{lll}
x & \text { if } & x \in[0,1] \\
2 x-1 & \text { if } & x \in\{1,2,3, \ldots\}
\end{array}\right.
$$

and $\alpha, \beta: X \rightarrow[0, \infty)$ by

$$
\alpha(x)=\left\{\begin{array}{ll}
0 & \text { if } x \in[0,1] \\
\frac{x+1}{2} & \text { if } x \in\{1,2,3, \ldots\}
\end{array} \text { and } \beta(x)= \begin{cases}0 & \text { if } x \in[0,1] \\
\frac{x+2}{3} & \text { if } x \in\{1,2,3, \ldots\}\end{cases}\right.
$$

Now, we show that $f$ is cyclic $(\alpha, \beta)$-admissible mapping. Since for any $x \in X$

$\alpha(x) \geq 1 \Leftrightarrow x \in\{1,2,3, \ldots\}$, we have $\beta(f x)=\beta(2 x-1)=\frac{2 x+1}{3} \geq 1$ for all $x \in\{1,2,3, \ldots\}$. Also, for any $x \in X \beta(x) \geq 1 \Leftrightarrow x \in\{1,2,3, \ldots\}$, we have $\alpha(f x)=\alpha(2 x-1)=\frac{2 x}{2}=x \geq 1$ for all $x \in\{1,2,3, \ldots\}$.

Therefore, $f$ is a cyclic $(\alpha, \beta)$-admissible mapping.

For $x, y \in X$ with $\alpha(x) \beta(x) \geq 1 \Longleftrightarrow x, y \in\{1,2,3, \ldots\}$, which implies that $f x=2 x-1$ and $f y=2 y-1$, therefore $f x$ and $f y$ are odd, and hence $d(f x, f y)=d(2 x-1,2 y-1)=5$ for all $x, y \in\{1,2,3, \ldots\}$.

We choose $\psi(t)=t, \varphi(t)=\frac{3 t}{4}, t \geq 0$.

Now, we consider the following cases to show that $f$ is almost generalized $(\alpha, \beta)-(\psi, \varphi)$ - contractive mapping with $L=\frac{25}{4}$. 
$\underline{\text { Case }}(\mathrm{i}): x, y \in\{1,3,5, \ldots\}$.

In this case,

$$
\begin{aligned}
& M_{s}(x, y)=\max \left\{d(x, y), d(x, f x), d(y, f y), \frac{d(x, f y)+d(y, f x)}{2\left(\frac{5}{4}\right)}\right\} \\
& =\max \left\{5,5,5,2\left(\frac{5+5}{5}\right)\right\}=\max \{5,4\}=5, \\
& M^{\prime}(x, y)=\max \{d(x, y), d(y, f y)\}=\max \{5,5\}=5 \text {, and } \\
& N(x, y)=\min \{d(x, f x), d(y, f x)\}=\min \{5,5\}=5 \text {. } \\
& \psi\left(s^{3} d(f x, f y)\right)=\psi\left(\left(\frac{5}{4}\right)^{3} d(2 x-1,2 y-1)\right)=\psi\left(\frac{625}{64}\right)=\frac{625}{64} \\
& \leq 5-\left(\frac{3}{4}\right) 5+\left(\frac{25}{4}\right) 5 \\
& \leq \psi\left(M_{s}(x, y)\right)-\varphi\left(M^{\prime}(x, y)\right)+L N(x, y)
\end{aligned}
$$

where $L=\frac{25}{4}$.

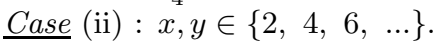

Here

$$
\begin{aligned}
M_{s}(x, y) & =\max \left\{d(x, y), d(x, f x), d(y, f y), \frac{d(x, f y)+d(y, f x)}{2\left(\frac{5}{4}\right)}\right\} \\
& =\max \left\{\left|\frac{1}{x}-\frac{1}{y}\right|, 2,2,2\left(\frac{2+2}{5}\right)\right\}=\max \left\{\left|\frac{1}{x}-\frac{1}{y}\right|, 2, \frac{8}{5}\right\}=2,
\end{aligned}
$$

$M^{\prime}(x, y)=\max \{d(x, y), d(y, f y)\}=\max \left\{\left|\frac{1}{x}-\frac{1}{y}\right|, 2\right\}=2$, and

$N(x, y)=\min \{d(x, f x), d(y, f x)\}=\min \{2,2\}=2$.

Now, we have

$$
\begin{aligned}
\psi\left(s^{3} d(f x, f y)\right) & =\psi\left(\left(\frac{5}{4}\right)^{3} d(2 x-1,2 y-1)\right)=\psi\left(\frac{625}{64}\right)=\frac{625}{64} \leq 2-\left(\frac{3}{4}\right) 2+\left(\frac{25}{4}\right) 2 \\
& \leq \psi\left(M_{s}(x, y)\right)-\varphi\left(M^{\prime}(x, y)\right)+L N(x, y)
\end{aligned}
$$

where $L=\frac{25}{4}$.

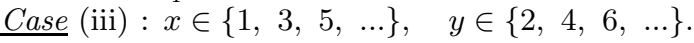

Here

$$
\begin{aligned}
M_{s}(x, y) & =\max \left\{d(x, y), d(x, f x), d(y, f y), \frac{d(x, f y)+d(y, f x)}{2\left(\frac{5}{4}\right)}\right\} \\
& =\max \left\{2,5,2,2\left(\frac{5+2}{5}\right)\right\}=\max \left\{5,2, \frac{14}{5}\right\}=5,
\end{aligned}
$$

$M^{\prime}(x, y)=\max \{d(x, y), d(y, f y)\}=\max \{2,2\}=2$, and

$N(x, y)=\min \{d(x, f x), d(y, f x)\}=\min \{5,2\}=2$.

Now, we have

$$
\begin{aligned}
\psi\left(s^{3} d(f x, f y)\right) & =\psi\left(\left(\frac{5}{4}\right)^{3} d(2 x-1,2 y-1)\right)=\psi\left(\frac{625}{64}\right)=\frac{625}{64} \\
& \leq 5-\left(\frac{3}{4}\right) 2+\left(\frac{25}{4}\right) 2 \\
& \leq \psi\left(M_{s}(x, y)\right)-\varphi\left(M^{\prime}(x, y)\right)+L N(x, y)
\end{aligned}
$$


where $L=\frac{25}{4}$

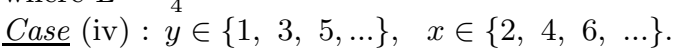

In this case

$M_{s}(x, y)=\max \left\{2,2,5,2\left(\frac{5+2}{5}\right)\right\}=5$,

$M^{\prime}(x, y)=\max \{d(x, y), d(y, f y)\}=\max \{2,5\}=5$, and

$N(x, y)=\min \{d(x, f y), d(y, f x)\}=\min \{2,5\}=2$.

Now, we have

$$
\begin{aligned}
\psi\left(s^{3} d(f x, f y)\right) & =\psi\left(\left(\frac{5}{4}\right)^{3} d(2 x-1,2 y-1)\right)=\psi\left(\frac{625}{64}\right) \\
& \leq \psi\left(M_{s}(x, y)\right)-\varphi\left(M^{\prime}(x, y)\right)+L N(x, y),
\end{aligned}
$$

as in the case (iii).

Hence, from all the above cases $f$ is an almost generalized $(\alpha, \beta)-(\psi, \varphi)$-contractive mapping. Here we observe that if $L=0$ then for any $x, y \in\{1,3,5, \ldots\}$

$$
\begin{aligned}
\psi\left(s^{3} d(f x, f y)\right) & =\psi\left(\left(\frac{5}{4}\right)^{3} d(2 x-1,2 y-1)\right)=\psi\left(\frac{625}{64}\right) \not \leq \psi(5) \\
& \not \leq \psi(5)-\varphi(5)=\psi\left(M_{s}(x, y)\right)-\varphi\left(M_{s}(x, y)\right)
\end{aligned}
$$

for any $\psi, \varphi \in \Psi$, so that $f$ is not an $(\alpha, \beta)-(\psi, \varphi)$ - contractive mapping.

Hence the class of 'almost generalized $(\alpha, \beta)-(\psi, \varphi)$ - contractive maps' is larger than 'the class of $(\alpha, \beta)-(\psi, \varphi)$ - contractive maps'.

Further, we observe that the metric $d$ defined in this example is not a metric in the usual sense for, by choosing $x=1, y=2$ and $z=3$, we have $d(x, y)=5 \not \leq 2+2=d(x, z)+d(z, y)$.

We state the following lemma which is useful to prove our main results.

Lemma 2.2. [13] Suppose $(X, d)$ is a $b$-metric space and $\left\{x_{n}\right\}$ be a sequence in $X$ such that $d\left(x_{n}, x_{n+1}\right) \rightarrow 0$ as $n \rightarrow \infty$. If $\left\{x_{n}\right\}$ is not a Cauchy sequence then there exists an $\epsilon>0$ and sequences of positive integers $\left\{m_{k}\right\}$ and $\left\{n_{k}\right\}$ with $n_{k}>m_{k} \geq k$ such that $d\left(m_{k}, n_{k}\right) \geq \epsilon$. For each $k>0$, corresponding to $m_{k}$, we can choose $n_{k}$ to be the smallest positive integer such that $d\left(x_{m_{k}}, x_{n_{k}}\right) \geq \epsilon, d\left(x_{m_{k}}, x_{n_{k}-1}\right)<\epsilon$ and

(i) $\epsilon \leq \limsup _{k \rightarrow \infty} d\left(x_{m_{k}}, x_{n_{k}}\right) \leq s \epsilon$

(ii) $\frac{\epsilon}{s} \leq \liminf _{k \rightarrow \infty} d\left(x_{m_{k}}, x_{n_{k}+1}\right) \leq \limsup _{k \rightarrow \infty} d\left(x_{m_{k}}, x_{n_{k}+1}\right) \leq s^{2} \epsilon$

(iii) $\frac{\epsilon}{s} \leq \liminf _{k \rightarrow \infty} d\left(x_{m_{k}+1}, x_{n_{k}}\right) \leq \limsup _{k \rightarrow \infty} d\left(x_{m_{k}+1}, x_{n_{k}}\right) \leq s^{2} \epsilon$

(iv) $\frac{\epsilon}{s^{2}} \leq \liminf _{k \rightarrow \infty} d\left(x_{m_{k}+1}, x_{n_{k}+1}\right) \leq \limsup _{k \rightarrow \infty} d\left(x_{m_{k}+1}, x_{n_{k}+1}\right) \leq s^{3} \epsilon$.

\section{Main results}

Theorem 3.1. Let $(X, d)$ be a complete $b$-metric space with the coefficient $s \geq 1$. Let $f: X \rightarrow X$ be a selfmapping of $X$. Assume that there exist two mappings $\alpha, \beta: X \rightarrow[0, \infty)$ and $\psi, \varphi \in \Psi$ such that $f$ is an almost generalized $(\alpha, \beta)-(\psi, \varphi)$ - contractive mapping. 
Further, suppose that

(1) there exists $x_{0} \in X$ such that $\alpha\left(x_{0}\right) \geq 1$ and $\beta\left(x_{0}\right) \geq 1$,

(2) $f$ is continuous,

(3) $f$ cyclic $(\alpha, \beta)$ - admissible mapping.

Then the sequence $\left\{x_{n}\right\}$ in $X$ defined by $x_{n+1}=f x_{n}, n=0,1,2, \ldots$, where $x_{0} \in X$ is given as in (1) is $b$-Cauchy and it is $b$-convergent to $z$ (say) in $X$, and $z$ is a fixed point of $f$.

Proof. By (1) we have $x_{0} \in X$ such that $\alpha\left(x_{0}\right) \geq 1$ and $\beta\left(x_{0}\right) \geq 1$,

Now, we define an iterative sequence $\left\{x_{n}\right\}$ by $x_{n+1}=f x_{n}$ for $n=0,1,2, \ldots$. If $x_{n_{0}+1}=x_{n_{0}}$ for some $n_{0} \in \mathbf{N} \cup\{0\}$, we have $f x_{n_{0}}=x_{n_{0}+1}=x_{n_{0}}$, so that $x_{n_{0}}$ is a fixed point of $f$ and we are through.

Hence, without loss of generality, we assume that $x_{n+1} \neq x_{n}$ for all $n \in \mathbf{N} \cup\{0\}$. Since $\alpha\left(x_{0}\right) \geq 1$ and $f$ is cyclic $(\alpha, \beta)$-admissible mapping, we have $\beta\left(x_{1}\right)=\beta\left(f x_{0}\right) \geq 1$, and this implies that $\alpha\left(x_{2}\right)=\alpha\left(f x_{1}\right) \geq 1$. By continuing this process, we obtain

$$
\alpha\left(x_{2 k}\right) \geq 1 \text { and } \beta\left(x_{2 k+1}\right) \geq 1 \text { for all } k \in \mathbf{N} \cup\{0\} .
$$

Since, $\beta\left(x_{0}\right) \geq 1$ and $f$ is a cyclic $(\alpha, \beta)$-admissible mapping, we have $\alpha\left(x_{1}\right)=\alpha\left(f x_{0}\right) \geq 1$ and this implies that $\beta\left(x_{2}\right)=\beta\left(f x_{1}\right) \geq 1$. On continuing this process, we obtain

$$
\beta\left(x_{2 k}\right) \geq 1 \text { and } \alpha\left(x_{2 k+1}\right) \geq 1 \text { for all } k \in \mathbf{N} \cup\{0\}
$$

Therefore, from (3.1) and (3.2) we have $\alpha\left(x_{n}\right) \geq 1$ and $\beta\left(x_{n}\right) \geq 1$ for all $n \in \mathbf{N} \cup\{0\}$.

First we claim that $\lim _{n \rightarrow \infty} d\left(x_{n}, x_{n+1}\right)=0$.

Since $\alpha\left(x_{n}\right) \beta\left(x_{n+1}\right) \geq 1$ for all $n \in \mathbf{N} \cup\{0\}$, from (2.4), we have

$$
\begin{aligned}
\psi\left(s^{3} d\left(f x_{n}, f x_{n+1}\right)\right) \leq \psi\left(M_{s}\left(x_{n}, x_{n+1}\right)\right)- & \varphi\left(M^{\prime}\left(x_{n}, x_{n+1}\right)\right) \\
& +L N\left(x_{n}, x_{n+1}\right)
\end{aligned}
$$

where

$$
\begin{aligned}
& M_{s}\left(x_{n}, x_{n+1}\right)=\max \left\{d\left(x_{n}, x_{n+1}\right), d\left(x_{n}, f x_{n}\right), d\left(x_{n+1}, f x_{n+1}\right), \frac{d\left(x_{n}, f x_{n+1}\right)+d\left(x_{n+1}, f x_{n}\right)}{2 s}\right\} \\
& =\max \left\{d\left(x_{n}, x_{n+1}\right), d\left(x_{n+1}, x_{n+2}\right), \frac{d\left(x_{n}, x_{n+2}\right)}{2 s}\right\} \\
& =\max \left\{d\left(x_{n}, x_{n+1}\right), d\left(x_{n+1}, x_{n+2}\right)\right\} \\
& \begin{aligned}
M^{\prime}\left(x_{n}, x_{n+1}\right) & =\max \left\{d\left(x_{n}, x_{n+1}\right), d\left(x_{n+1}, f x_{n+1}\right)\right\}=\max \left\{d\left(x_{n}, x_{n+1}\right), d\left(x_{n+1}, x_{n+2}\right)\right\} \\
\text { and } & \\
N\left(x_{n}, x_{n+1}\right)= & \min \left\{d\left(x_{n}, f x_{n}\right), d\left(x_{n+1}, f x_{n}\right)\right\} \\
& =\min \left\{d\left(x_{n}, x_{n+1}\right), d\left(x_{n+1}, x_{n+1}\right)\right\} \\
& =\min \left\{d\left(x_{n}, x_{n+1}\right), 0\right\}=0 .
\end{aligned}
\end{aligned}
$$


Now, if $d\left(x_{n}, x_{n+1}\right)<d\left(x_{n+1}, x_{n+2}\right)$ for some $n \in \mathbb{N} \cup\{0\}$, it follows from (3.3) that

$$
\begin{aligned}
\psi\left(d\left(x_{n+1}, x_{n+2}\right)\right) & =\psi\left(d\left(f x_{n}, f x_{n+1}\right)\right) \\
& \leq \psi\left(s^{3} d\left(f x_{n}, f x_{n+1}\right)\right) \\
& \leq \psi\left(d\left(x_{n+1}, x_{n+2}\right)\right)-\varphi\left(d\left(x_{n+1}, x_{n+2}\right)\right)+L(0) \\
& =\psi\left(d\left(x_{n+1}, x_{n+2}\right)\right)-\varphi\left(d\left(x_{n+1}, x_{n+2}\right)\right) \\
& <\psi\left(d\left(x_{n+1}, x_{n+2}\right)\right),
\end{aligned}
$$

a contradiction.

Hence

$d\left(x_{n}, x_{n+1}\right) \geq d\left(x_{n+1}, x_{n+2}\right)$ for all $n \in \mathbf{N} \cup\{0\}$.

Therefore we have

$$
\begin{aligned}
\psi\left(d\left(x_{n+1}, x_{n+2}\right)\right) & =\psi\left(d\left(f x_{n}, f x_{n+1}\right)\right) \\
& \leq \psi\left(s^{3} d\left(f x_{n}, f x_{n+1}\right)\right) \\
& \leq \psi\left(d\left(x_{n}, x_{n+1}\right)\right)-\varphi\left(d\left(x_{n}, x_{n+1}\right)\right) \\
& <\psi\left(d\left(x_{n}, x_{n+1}\right)\right) .
\end{aligned}
$$

Since $\psi$ is nondecreasing, the sequence $\left\{d\left(x_{n}, x_{n+1}\right)\right\}$ is decreasing and bounded from below. Thus there exists $r \geq 0$ such that $\lim _{n \rightarrow \infty} d\left(x_{n}, x_{n+1}\right)=r$. Suppose $r>0$. Hence we have

$$
\begin{aligned}
\psi\left(d\left(x_{n+1}, x_{n+2}\right)\right) & =\psi\left(d\left(f x_{n}, f x_{n+1}\right)\right) \\
& \leq \psi\left(s^{3} d\left(f x_{n}, f x_{n+1}\right)\right) \\
& \leq \psi\left(d\left(x_{n}, x_{n+1}\right)\right)-\varphi\left(d\left(x_{n}, x_{n+1}\right)\right)+\theta(0) \\
& =\psi\left(d\left(x_{n}, x_{n+1}\right)\right)-\varphi\left(d\left(x_{n}, x_{n+1}\right)\right) .
\end{aligned}
$$

On letting $n \rightarrow \infty$ and using the continuity of $\psi$ and $\varphi$ in (3.4), we have $\left.\psi(r) \leq \psi\left(s^{3} r\right) \leq \psi(r)\right)-\varphi(r)<\psi(r)$, a contradiction.

Hence $r=0$, i.e., $\lim _{n \rightarrow \infty} d\left(x_{n}, x_{n+1}\right)=0$.

We now prove that $\left\{x_{n}\right\}$ is a b-Cauchy sequence. Suppose $\left\{x_{n}\right\}$ is not a b-Cauchy sequence. Then by Lemma 2.2 there exist $\epsilon>0$ and sequences of positive integers $\left\{n_{k}\right\}$ and $\left\{m_{k}\right\}$ with $n_{k}>m_{k}>k$ such that $d\left(x_{m_{k}}, x_{n_{k}}\right) \geq \epsilon$, $d\left(x_{m_{k}}, x_{n_{k}-1}\right)<\epsilon$ and (i) - (iv) of Lemma 2.2 hold.

Since $\alpha\left(x_{m_{k}}\right) \geq 1$ and $\beta\left(x_{n_{k}}\right) \geq 1$ which implies that $\alpha\left(x_{m_{k}}\right) \beta\left(x_{n_{k}}\right) \geq 1$. Now, from (2.4) we have

$$
\begin{aligned}
\psi\left(d\left(x_{m_{k}+1}, f x_{n_{k}+1}\right)\right) & =\psi\left(d\left(f x_{m_{k}}, f x_{n_{k}}\right)\right) \\
\leq & \psi\left(s^{3} d\left(f x_{m_{k}}, f x_{n_{k}}\right)\right) \\
\leq & \psi\left(M_{s}\left(x_{m_{k}}, x_{n_{k}}\right)-\varphi\left(M^{\prime}\left(x_{m_{k}}, x_{n_{k}}\right)\right)\right. \\
& +L N\left(x_{m_{k}}, x_{n_{k}}\right),
\end{aligned}
$$

where

$M_{s}\left(x_{m_{k}}, x_{n_{k}}\right)=\max \left\{d\left(x_{m_{k}}, x_{n_{k}}\right), d\left(x_{m_{k}}, f x_{m_{k}}\right), d\left(x_{n_{k}}, f x_{n_{k}}\right), \frac{d\left(f x_{m_{k}}, x_{n_{k}}\right)+d\left(x_{m_{k}}, f x_{n_{k}}\right)}{2 s}\right\}$, 
$M^{\prime}\left(x_{m_{k}}, x_{n_{k}}\right)=\max \left\{d\left(x_{m_{k}}, x_{n_{k}}\right), d\left(x_{n_{k}}, f x_{n_{k}}\right)\right\}$, and

$N\left(x_{m_{k}}, x_{n_{k}}\right)=\min \left\{d\left(x_{m_{k}}, f x_{m_{k}}\right), d\left(x_{n_{k}}, f x_{m_{k}}\right)\right\}$.

On taking Limit supremum as $n \rightarrow \infty$, we have

$$
\begin{aligned}
& \epsilon \leq \limsup _{k \rightarrow \infty} M_{s}\left(x_{m_{k}}, x_{n_{k}}\right) \leq \max \left\{s \epsilon, 0, \frac{s^{2} \epsilon+s^{2} \epsilon}{2 s}\right\}=s \epsilon, \\
& \epsilon \leq \limsup _{k \rightarrow \infty} M^{\prime}\left(x_{m_{k}}, x_{n_{k}}\right) \leq \max \{s \epsilon, 0,\}=s \epsilon, \text { and } \\
& \limsup _{k \rightarrow \infty} N\left(x_{m_{k}}, x_{n_{k}}\right) \leq \max \{s \epsilon, 0,\}=0 .
\end{aligned}
$$

Also on taking limit infimum as $k \rightarrow \infty$, we have

$\epsilon \leq \liminf _{k \rightarrow \infty} M^{\prime}\left(x_{m_{k}}, x_{n_{k}}\right) \leq \limsup _{k \rightarrow \infty} M^{\prime}\left(x_{m_{k}}, x_{n_{k}}\right) \leq \max \{s \epsilon, 0\}=s \epsilon$.

Now, using (3.5), we have

$$
\begin{aligned}
\psi(s \epsilon)=\psi\left(s^{3} \frac{\epsilon}{s^{2}}\right) & \leq \psi\left(s^{3} \limsup _{k \rightarrow \infty} d\left(x_{m_{k}+1}, x_{n_{k}+1}\right)\right) \\
& =\psi\left(s^{3} \limsup _{k \rightarrow \infty} d\left(f x_{m_{k}}, f x_{n_{k}}\right)\right. \\
& \leq \psi\left(\limsup _{k \rightarrow \infty} M_{s}\left(x_{m_{k}}, x_{n_{k}}\right)-\varphi\left(\liminf _{k \rightarrow \infty} M^{\prime}\left(x_{m_{k}}, x_{n_{k}}\right)\right)\right. \\
& \left.+L \limsup _{k \rightarrow \infty} N\left(x_{m_{k}}, x_{n_{k}}\right)\right) \\
& \leq \psi(s \epsilon)-\varphi(\epsilon) \\
& <\psi(s \epsilon),
\end{aligned}
$$

a contradiction. So we conclude that $\left\{x_{n}\right\}$ is a $b$-Cauchy sequence in $(X, d)$.

Since $(X, d)$ is $b$-complete, it follows that there exists $z \in X$ such that

$\lim _{n \rightarrow \infty} x_{n}=z$.

Since $f$ is continuous, we have $\lim _{n \rightarrow \infty} f x_{n}=f z$, and

$f z=\lim _{n \rightarrow \infty} f x_{n}=\lim _{n \rightarrow \infty} x_{n+1}=z$.

Theorem 3.2. Let $(X, d)$ be a complete $b$-metric space with the coefficient $s \geq 1$. Let $f: X \rightarrow X$ be a selfmapping of $X$. Assume that there exist two mappings $\alpha, \beta: X \rightarrow[0, \infty)$ and $\psi, \varphi \in \Psi$ such that $f$ is an almost generalized $(\alpha, \beta)-(\psi, \varphi)$ - contractive mapping.

Further, suppose that

(1) there exists $x_{0} \in X$ such that $\alpha\left(x_{0}\right) \geq 1$ and $\beta\left(x_{0}\right) \geq 1$,

(2) $f$ cyclic $(\alpha, \beta)$ - admissible mapping,

(3) If $\left\{x_{n}\right\}$ is a sequence in $X$ such that $x_{n} \rightarrow z$ and $\beta\left(x_{n}\right) \geq 1$ for all $n$, then $\beta(z) \geq 1$.

Then $f$ has a fixed point.

Proof. From the similar arguments as in the proof of Theorem 3.1 we obtain the sequence $\left\{x_{n}\right\}$ is Cauchy and $\beta\left(x_{n}\right) \geq 1$ for all $n \in \mathbb{N} \cup\{0\}$. Since $(X, d)$ is 
$b$ - complete $b$ - metric space, there exists $z \in X$ such that $x_{n} \rightarrow z$ as $n \rightarrow \infty$. From (3) we have $\beta(z) \geq 1$.

We assume that $f z \neq z$. From the triangular inequality, we have $d(z, f z) \leq s\left[d\left(z, f x_{n}\right)+d\left(f x_{n} f z\right)\right]$.

On taking the limit supremum as $n \rightarrow \infty$, we have

$$
\frac{1}{s} d(z, f z) \leq \limsup _{n \rightarrow \infty} d\left(f x_{n}, f z\right) .
$$

Also we have $d\left(f x_{n}, f z\right) \leq s\left[d\left(f x_{n}, z\right)+d(z f z)\right]$.

On taking the limit supremum as $n \rightarrow \infty$, we have

$$
\limsup _{n \rightarrow \infty} d\left(f x_{n}, f z\right) \leq s d(z, f z) .
$$

From (3.7) and (3.8), we have

$$
\frac{1}{s} d(z, f z) \leq \limsup _{n \rightarrow \infty} d\left(f x_{n}, f z\right) \leq s d(z, f z) .
$$

From (2.4), we have

$$
\begin{aligned}
\psi(d(z, f z)) \leq \psi\left(s^{2} d(z, f z)\right)= & \psi\left(s^{3}\left[\frac{1}{s} d(z, f z)\right]\right) \\
\leq & \psi\left(s^{3}\left[\limsup _{n \rightarrow \infty} d\left(f x_{n}, f z\right)\right]\right) \\
= & \limsup _{n \rightarrow \infty} \psi\left(s^{3}\left[d\left(f x_{n}, f z\right)\right]\right) \\
\leq & \limsup _{n \rightarrow \infty}\left[\psi\left(M_{s}\left(x_{n}, z\right)\right)-\varphi\left(M^{\prime}\left(x_{n}, z\right)\right)\right. \\
& \left.+L N\left(x_{n}, z\right)\right] .
\end{aligned}
$$

Hence we have

$$
\begin{aligned}
\psi(d(z, f z)) \leq \psi\left(s^{2} d(z, f z)\right) \leq & \limsup _{n \rightarrow \infty} \psi\left(M_{s}\left(x_{n}, z\right)\right) \\
& +\limsup _{n \rightarrow \infty}\left(-\varphi\left(M^{\prime}\left(x_{n}, z\right)\right)\right) \\
& +L \limsup _{n \rightarrow \infty} N\left(x_{n}, z\right),
\end{aligned}
$$

where

$d(z, f z) \leq M_{s}\left(x_{n}, z\right)=\max \left\{d\left(x_{n}, z\right), d\left(x_{n}, f x_{n}\right), d(z, f z), \frac{d\left(x_{n}, f z\right)+d\left(z, f x_{n}\right)}{2 s}\right\}$, $d(z, f z) \leq M^{\prime}\left(x_{n}, z\right)=\max \left\{d\left(x_{n}, z\right), d(z, f z)\right\}$,

$N\left(x_{n}, z\right)=\min \left\{d\left(x_{n}, f x_{n}\right), d\left(z, f x_{n}\right)\right\}$.

On taking the limits of $M_{s}\left(x_{n}, z\right), M^{\prime}\left(x_{n}, z\right)$ and $N\left(x_{n}, z\right)$ as $n \rightarrow \infty$ and using (3.9), we have 
$d(z, f z) \leq \lim _{n \rightarrow \infty} M_{s}\left(x_{n}, z\right)=\max \left\{0,0, d(z, f z), \limsup _{n \rightarrow \infty} \frac{d\left(x_{n}, f z\right)}{2 s}\right\}=d(z, f z)$,

$\lim _{n \rightarrow \infty} M^{\prime}\left(x_{n}, z\right)=\max \{0, d(z, f z)\}=d(z, f z)$,

$\limsup _{k \rightarrow \infty} N\left(x_{n}, z\right)=\{0,0\}=$,0 .

$k \rightarrow \infty$

From (3.11) we have

$$
\begin{aligned}
\psi(d(z, f z)) & \leq \psi(d(z, f z))-\varphi(d(z, f z)) \\
& <\psi(d(z, f z))
\end{aligned}
$$

a contradiction. Hence $f z=z$.

Theorem 3.3. In addition to the hypothesis of Theorem 3.1 (Theorem 3.2), if $\alpha(u) \geq 1$ or $\beta(u) \geq 1$ whenever $f u=u$. Then $f$ has a unique fixed point.

Proof. Suppose that $u$ and $w$ be two fixed points of $f$ with $u \neq w$, that is, $f u=u$ and $f w=w$. By the hypothesis we have $\alpha(u) \geq 1$ or $\beta(u) \geq 1$ and $\alpha(w) \geq 1$ or $\beta(w) \geq 1$. Since $f$ is a cyclic $(\alpha, \beta)$-admissible mapping, we have $\alpha(u) \geq 1 \Longrightarrow \beta(u)=\beta(f u) \geq 1$, and $\beta(u) \geq 1 \Longrightarrow \alpha(u)=\alpha(f u) \geq 1$, Therefore we have $\beta(u) \geq 1$ and $\alpha(u) \geq 1$. And also $\alpha(w) \geq 1 \Longrightarrow \beta(w)=\beta(f w) \geq 1$, and $\beta(w) \geq 1 \Longrightarrow \alpha(w)=\alpha(f w) \geq 1$. then we have $\beta(w) \geq 1$ and $\alpha(w) \geq 1$. Hence we have $\alpha(w) \geq 1, \alpha(u) \geq 1, \beta(w) \geq 1$ and $\beta(u) \geq 1$ this implies $\alpha(u) \beta(w) \geq 1$.

Now, from (2.4) we have

$$
\begin{aligned}
\psi(d(u, w)) & =\psi(d(f u, f w)) \leq \psi\left(s^{3} d(f u, f w)\right) \\
& \leq \psi\left(M_{s}(u, w)\right)-\varphi\left(M^{\prime}(u, u)\right)+L N(u, w)
\end{aligned}
$$

Where

$$
\begin{aligned}
M_{s}(u, w) & =\max \left\{d(u, w), d(u, f u), d(w, f w), \frac{d(u, f w)+d(w, f u)}{2 s}\right\} \\
& =\max \left\{d(u, w), d(u, u), d(w, w), \frac{d(u, w)+d(w, u)}{2 s}\right\} \\
& =\max \left\{d(u, w), 0, \frac{d(u, w)}{s}\right\} \\
& =d(u, w)
\end{aligned}
$$

$M^{\prime}(u, w)=\max \{d(u, w), d(w, f w)\}=\max \{d(u, w), d(w, w)\}=\max \{d(u, w), 0\}=d(u, w)$, $N(u, w)=\min \{d(u, f u), d(w, f u)\}=\min \{d(u, u), d(w, u)\}=\min \{0, d(w, u)\}=0$. by using the inequality (3.13), we have

$$
\begin{aligned}
\psi(d(u, w)) & =\psi(d(f u, f w)) \leq \psi\left(s^{3} d(f u, f w)\right) \\
& \leq \psi\left(M_{s}(u, w)\right)-\varphi\left(M^{\prime}(u, u)\right)+L N(u, w) \\
& =\psi(d(u, w))-\varphi(d(u, w))+L \theta(0) \\
& =\psi(d(u, w))-\varphi(d(u, w)) \\
& <\psi(d(u, w))
\end{aligned}
$$


a contradiction. Therefore $u=w$

Hence $f$ has a unique fixed point.

Definition 3.1. Let $(X, d)$ be a $b$-metric space with coefficient $s \geq 1$, and $A$ and $B$ be two closed subsets of $X$ such that $A \cap B \neq \varnothing$. Let $f: A \cup B \rightarrow A \cup B$ be a mapping. If there exist $\psi, \varphi \in \Psi$ and $L \geq 0$ such that

$$
\psi\left(s^{3} d(f x, f y)\right) \leq \psi\left(M_{s}(x, y)\right)-\varphi\left(M^{\prime}(x, y)\right)+L N(x, y),
$$

for all $x \in A$ and $y \in B$. Then we say that $f$ is an almost generalized $(A, B)-(\psi, \varphi)$-contractive mapping.

Theorem 3.4. Let $A$ and $B$ be two nonempty closed subsets of a complete $b$-metric space $(X, d)$ such that $A \cap B \neq \varnothing$, and let $f: A \cup B \rightarrow A \cup B$ be a cyclic mapping. If $f$ is an almost generalized $(A, B)-(\psi, \varphi)$-contractive mapping, then $f$ has a unique fixed point in $A \cap B$.

Proof. Let us define $\alpha, \beta: A \cup B \rightarrow A \cup B$ by

$\alpha(x)=\left\{\begin{array}{ll}1 & \text { if } x \in A \\ 0 & \text { otherwise }\end{array} \quad \beta(x)=\left\{\begin{array}{cc}1 & \text { if } x \in B \\ 0 & \text { otherwise }\end{array}\right.\right.$

For any $x, y \in A \cup B$ with $\alpha(x) \beta(y) \geq 1$, this implies that $x \in A$ and $y \in B$, then from the hypothesis we have

$\psi\left(s^{3} d(f x, f y)\right) \leq \psi\left(M_{s}(x, y)\right)-\varphi\left(M^{\prime}(x, y)\right)+L N(x, y)$.

Thus, inequality (3.16) holds. Therefore $f$ is an almost generalized

$(\alpha, \beta)-(\psi, \varphi)$-contractive mapping.

Since $A \cap B \neq \varnothing$ there exists $x_{0} \in A \cap B$ this implies that $x_{0} \in A$ and $x_{0} \in B$ hence $\alpha\left(x_{0}\right) \geq 1$ and $\beta\left(x_{0}\right) \geq 1$.

Let $\left\{x_{n}\right\}$ be a sequence in $X$ such that $\beta\left(x_{n}\right) \geq 1$ for all $n \in \mathbb{N} \cup\{0\}$ and $x_{n} \rightarrow x$ as $n \rightarrow \infty$, then $x_{n} \in B$ for all $n \in \mathbb{N} \cup\{0\}$. Since $B$ is closed we have $x \in B$ hence $\beta(x) \geq 1$.

Therefore all hypotheses of Theorem 3.2 hold.

Hence $f$ has a fixed point. Let $u$ (say) be the fixed point of $f$. If $u \in A$, then $u=f u \in B$. Similarly, if $u \in B$, then $u=f u \in A$.

Hence $u \in A \cap B$. And also $\alpha(u) \geq 1$ and $\beta(u) \geq 1$. Therefore, by Theorem 3.3, $f$ has a unique fixed point.

\section{Corollaries and examples}

Corollary 4.1. Let $(X, d)$ be a complete $b$-metric space with the coefficient $s \geq 1$ and $f: X \rightarrow X$ be a continuous selfmaping of $X$. If there exist $\psi, \varphi \in \Psi$ such that $\psi\left(s^{3} d(f x, f y)\right) \leq \psi\left(M_{s}(x, y)\right)-\varphi\left(M^{\prime}(x, y)\right)+L N(x, y) \quad$ for all $x, y \in X$.

Then $f$ has a fixed point.

Proof. By choosing $\alpha(x)=\beta(x)=1$ in Theorem 3.1, the conclusion of this corollary follows. 
Corollary 4.2. Let $(X, d)$ be a complete $b$-metric space with the coefficient $s \geq 1$. Let $f: X \rightarrow X$ be a selfmapping of $X$. Assume that there exist two mappings $\alpha, \beta: X \rightarrow[0, \infty)$ and $\psi, \varphi \in \Psi$ such that for all $x, y \in X$ with $\alpha(x) \beta(y) \geq 1$

$\Longrightarrow \psi(d(f x, f y)) \leq \psi(M(x, y))-\varphi\left(M^{\prime}(x, y)\right)+L N(x, y)$.

Further, suppose that

(1) there exists $x_{0} \in X$ such that $\alpha\left(x_{0}\right) \geq 1$ and $\beta\left(x_{0}\right) \geq 1$,

(2) $f$ is continuous,

(3) $f$ cyclic $(\alpha, \beta)$ - admissible mapping.

Then the sequence $\left\{x_{n}\right\}$ in $X$ defined by $x_{n+1}=f x_{n}, n=0,1,2, \ldots$, where $x_{0} \in X$ is given as in (1) is $b$-Cauchy and it is b-convergent to $z$ (say) in $X$, and $z$ is a fixed point of $f$.

Proof. The result follows from Theorem 3.1 by taking $s=1$.

By choosing $s=1$ and $\alpha(x)=\beta(x)=1$ in Theorem 3.1, we have the following.

Corollary 4.3. Let $(X, d)$ be a complete metric space and $f: X \rightarrow X$ be selfmapping of $X$. If there exist $\psi, \varphi \in \Psi$ such that $\psi(d(f x, f y)) \leq \psi(M(x, y))-\varphi\left(M^{\prime}(x, y)\right)+L N(x, y) \quad$ for all $x, y \in X$. Then $f$ has a fixed point.

Remark 4.1. Here we observe that Theorem 2.1 is a corollary to Corollary 4.3. For any $x, y \in X$ we have

$$
\begin{aligned}
\psi(d(f x, f y)) & \leq \psi(d(x, y))-\varphi(d(x, y)) \leq \psi\left(M_{s}(x, y)\right)-\varphi\left(M^{\prime}{ }_{s}(x, y)\right) \\
& \leq \psi\left(M_{s}(x, y)\right)-\varphi\left(M^{\prime}(x, y)\right)+L N(x, y) .
\end{aligned}
$$

Corollary 4.4. (Theorem 2.4) Let $(X, d)$ be a complete $b$-metric space with the coefficient $s \geq 1$. Let $f: X \rightarrow X$ be a selfmapping of $X$. Assume that there exist two mappings $\alpha, \beta: X \rightarrow[0, \infty)$ and $\psi, \varphi \in \Psi$ such that for all $x, y \in X$ with $\alpha(x) \beta(y) \geq 1$

$\Longrightarrow \psi\left(s^{3} d(f x, f y)\right) \leq \psi\left(M_{s}(x, y)\right)-\varphi\left(M_{s}(x, y)\right)$

Further, suppose that

(1) there exists $x_{0} \in X$ such that $\alpha\left(x_{0}\right) \geq 1$ and $\beta\left(x_{0}\right) \geq 1$,

(2) $f$ is continuous,

(3) $f$ cyclic $(\alpha, \beta)$ - admissible mapping.

Then the sequence $\left\{x_{n}\right\}$ in $X$ defined by $x_{n+1}=f x_{n}, n=0,1,2, \ldots$, where $x_{0} \in X$ is given as in (1) is b-Cauchy and it is $b$-convergent to $z$ (say) in $X$, and $z$ is a fixed point of $f$.

Proof. By hypothesis, we have for all $x, y \in X$ with $\alpha(x) \beta(y) \geq 1$

$$
\begin{aligned}
\Longrightarrow \psi\left(s^{3} d(f x, f y)\right) & \leq \psi\left(M_{s}(x, y)\right)-\varphi\left(M_{s}(x, y)\right) \\
& \leq \psi\left(M_{s}(x, y)\right)-\varphi\left(M^{\prime}(x, y)\right) \\
& \leq \psi\left(M_{s}(x, y)\right)-\varphi\left(M^{\prime}(x, y)\right)+L N(x, y)
\end{aligned}
$$


Therefore, $f$ satisfies the inequality (2.4) with $L=0$. Hence by Theorem 3.1, $f$ has a fixed point

Corollary 4.5. Let $A$ and $B$ be two nonempty closed subsets of a complete metric space $(X, d)$ such that $A \cap B \neq \varnothing$, and let $f: A \cup B \rightarrow A \cup B$ be a cyclic mapping. If there exist $\psi, \varphi \in \Psi$ and $L \geq 0$ such that

$\psi(d(f x, f y)) \leq \psi\left(M_{s}(x, y)\right)-\varphi\left(M^{\prime}(x, y)\right)+L N(x, y)$, then $f$ has a unique fixed point in $A \cap B$.

Proof. By choosing $s=1$ in Theorem 3.4 the conclusion of the corollary follows.

Corollary 4.6. Let $A$ and $B$ be two nonempty closed subsets of a complete metric space $(X, d)$ such that $A \cap B \neq \varnothing$, and let $f: A \cup B \rightarrow A \cup B$ be a cyclic mapping. If there exist $\psi, \varphi \in \Psi$ such that

$\psi\left(s^{3} d(f x, f y)\right) \leq \psi\left(M_{s}(x, y)\right)-\varphi\left(M^{\prime}(x, y)\right)$, then $f$ has a unique fixed point in $A \cap B$.

Proof. follows from Theorem 3.4 by taking $L=0$.

Example 4.1. Let $X=[2,3] \cup\{4,5,6, \ldots\}$, we define $d: X \times X \rightarrow[0, \infty)$ by

$d(x, y)= \begin{cases}0 & \text { if } x=y \\ \frac{1}{x}+\frac{1}{y} & \text { if } x, y \in[2,3] \\ 4+\frac{1}{x}+\frac{1}{y} & \text { if } x, y \in\{4,5,6, \ldots\} \\ 2 & \text { otherwise. }\end{cases}$

Clearly, $d$ is a $b$-metric space with the coefficient $s \geq \frac{89}{80}$.

We define $f: X \rightarrow X$ by

$$
f(x)= \begin{cases}3-\frac{x}{4} & \text { if } x \in[2,3] \\ 2+\frac{3}{4 x} & \text { if } x \in\{3,4,5,6, \ldots\} .\end{cases}
$$

and $\alpha, \beta: X \rightarrow[0, \infty)$ by

$\alpha(x)=\left\{\begin{array}{ll}\frac{3}{x} & \text { if } x \in[2,3] \\ 0 & \text { otherwise, }\end{array} \quad \beta(x)= \begin{cases}\frac{4}{x} & \text { if } x \in[2,3] \\ 0 & \text { otherwise. }\end{cases}\right.$

Since for any $x \in X, \alpha(x) \geq 1 \Longleftrightarrow x \in[2,3]$, we have

$\beta(f x)=\frac{3}{f x}=\frac{3}{3-\frac{x}{4}} \geq 1$, and also $x \in X, \beta(x) \geq 1 \Longleftrightarrow x \in[2,3]$, we have

$\alpha(f x)=\frac{4}{f x}=\frac{4}{3-\frac{x}{4}} \geq 1$. Therefore, $f$ is cyclic $(\alpha, \beta)$ - admissible mapping.

Next, we show that $f$ is an almost generalized $(\alpha, \beta)-(\psi, \varphi)$ - contractive mapping.

For $x, y \in X$ with $\alpha(x) \beta(x) \geq 1 \Longleftrightarrow x, y \in[2,3]$. Hence, for $x, y \in[2,3]$ $f x=3-\frac{x}{4}$ and $f y=3-\frac{y}{4}$ and for $x \neq y$ we have

$$
\begin{aligned}
M_{s}(x, y) & =\max \left\{d(x, y), d(x, f x), d(y, f y), \frac{d(x, f y)+d(y, f x)}{2 s}\right\} \\
& =\max \left\{\frac{1}{x}+\frac{1}{y}, \frac{1}{x}+\frac{1}{f x}, \frac{1}{y}+\frac{1}{f y}, \frac{\frac{1}{x}+\frac{1}{f y}+\frac{1}{y}+\frac{1}{f x}}{2\left(\frac{89}{80}\right)}\right\} \geq \frac{2}{3},
\end{aligned}
$$


$M^{\prime}(x, y)=\max \left\{d(x, y), d(y, f y)=\max \left\{\frac{1}{x}+\frac{1}{y}, \frac{1}{y}+\frac{1}{f y}\right\} \leq 1\right.$, and $N(x, y)=\min \{d(x, f x), d(y, f x)\}=\min \left\{\frac{1}{y}+\frac{1}{f x}, \frac{1}{x}+\frac{1}{f x}\right\} \geq \frac{2}{5}$.

We now choose $\psi(t)=t, \varphi(t)=\frac{t}{4}, t \geq 0$ then, we have

$$
\begin{aligned}
\psi\left(s^{3} d(f x, f y)\right) & =d\left(\left(\frac{89}{80}\right)^{3} d\left(3-\frac{x}{4}, 3-\frac{y}{4}\right)\right) \\
& =\left(\frac{89}{80}\right)^{3}\left(\frac{1}{3-\frac{x}{4}}+\frac{1}{3-\frac{y}{4}}\right) \\
& \leq\left(\frac{89}{80}\right)^{3}\left(\frac{8}{9}\right) \\
& \leq \frac{2}{3}-\frac{1}{4}(1)+3\left(\frac{2}{5}\right) \\
& \leq \psi\left(M_{s}(x, y)-\varphi\left(M^{\prime}(x, y)\right)+L N(x, y) .\right.
\end{aligned}
$$

Hence $f$ is an almost generalized $(\alpha, \beta)-(\psi, \varphi)$ - contractive mapping with $L=3$.

Therefore, $f$ satisfies all the hypotheses of Theorem 3.1 and $x=\frac{12}{5}$ is a fixed point of $f$.

Here we observe that when $L=0$, the inequality (2.4) fails to hold for $x=2$ and $y=3, \quad$ we have

$$
\begin{aligned}
\psi\left(s^{3} d(f x, f y)\right) & =d\left(\left(\frac{89}{80}\right)^{3} d(f 2, f 3)\right)=\left(\frac{89}{80}\right)^{3}\left(\frac{38}{45}\right) \\
& \not \downarrow \psi\left(\frac{9}{10}\right)-\varphi\left(\frac{5}{6}\right) \\
& =\psi\left(M_{s}(2,3)-\varphi\left(M^{\prime}(2,3)\right)=\psi\left(M_{s}(x, y)-\varphi\left(M^{\prime}(x, y)\right) .\right.\right.
\end{aligned}
$$

for any $\psi$ and $\varphi$. Hence $f$ is not an $(\alpha, \beta)-(\psi, \varphi)$-contractive mapping. Therefore Theorem 2.4 is not applicable.

Further, this example shows the importance of $L$ in the inequality (2.4) of the almost generalized $(\alpha, \beta)-(\psi, \varphi)$ - contractive mapping.

Remark 4.2. From Example 4.1 and Corollary 4.4 we observe that Theorem 3.1(Theorem 3.2) is a generalization of Theorem 2.4.

Example 4.2. Let $X=\left\{0, \frac{1}{2}, \frac{1}{3}, \frac{1}{4}, \ldots\right\} \cup[1,2]$. We define $d: X \times X \rightarrow[0, \infty)$ by $d(x, y)= \begin{cases}0 & \text { if } x=y \\ |x-y| & \text { if } x, y \in\left\{0, \frac{1}{2}, \frac{1}{4}, \frac{1}{6}, \ldots\right\} \\ 6 & \text { if } x, y \in\left\{\frac{1}{3}, \frac{1}{5}, \frac{1}{7}, \ldots\right\} \text { and } x \neq y \\ 2 & \text { otherwise. }\end{cases}$

Then it is easy to see that $(X, d)$ is a $b$-metric space with the coefficient $s=\frac{3}{2}$. Now, we define $f: X \rightarrow X$ by

$$
f x=\left\{\begin{array}{l}
2-x \text { if } x \in\left\{0,1, \frac{1}{2}, \frac{1}{3}, \frac{1}{4}, \ldots\right\} \\
\frac{x}{2}+\frac{1}{2} \quad \text { if } x \in[1,2] .
\end{array}\right.
$$

and $\alpha, \beta: X \rightarrow[0, \infty)$ by

$$
\alpha(x)=\beta(x)=\left\{\begin{array}{l}
x \text { if } x \in[1,2] \\
0 \text { otherwise. }
\end{array} .\right.
$$


Since for any $x \in X, \alpha(x) \geq 1 \Longleftrightarrow x \in[1,2]$, we have

$\beta(f x)=\beta\left(\frac{x}{2}+\frac{1}{2}\right)=\frac{x}{2}+\frac{1}{2} \geq 1$. Since $\alpha(x)=\beta(x)$, clearly $f$ is a

cyclic $(\alpha, \beta)$-admissible mapping.

We choose $\psi(t)=t, \varphi(t)=\frac{t}{4}$ for $t \geq 0$.

Now, we show that $f$ is almost generalized $(\alpha, \beta)-(\psi, \varphi)$ - contractive mapping with $L=\frac{22}{8}$.

Since $\alpha(x) \beta(y) \geq 1 \Longleftrightarrow x, y \in[1,2]$, for $x, y \in[1,2]$, we have

$M_{s}(x, y)=\max \left\{d(x, y), d(x, f x), d(y, f y), \frac{d(x, f y)+d(y, f x)}{2\left(\frac{3}{2}\right)}\right\}=\left\{2, \frac{2+2}{2\left(\frac{3}{2}\right)}\right\}=2$,

$M^{\prime}(x, y)=\max \{d(x, y), d(y, f y)\}=\{2,2\}=2$ and

$N(x, y)=\operatorname{Min}\{d(x, f x), d(y, f x)\}=\{2,2\}=2$.

Hence, for $x, y \in[1,2]$, we have

$$
\begin{aligned}
\psi\left(s^{3} d(f x, f y)\right) & =\psi\left(\left(\frac{3}{2}\right)^{3} d\left(\frac{x}{2}+\frac{1}{2}, \frac{y}{2}+\frac{1}{2}\right)\right)=\psi\left(\frac{27}{8} 2\right)=\frac{27}{4} \\
& \leq 2-\frac{2}{4}+\left(\frac{22}{8}\right) 2 \\
& \leq \psi\left(M_{s}(x, y)\right)-\varphi\left(M^{\prime}(x, y)\right)+L N(x, y) .
\end{aligned}
$$

Hence $f$ is an almost generalized $(\alpha, \beta)-(\psi, \varphi)$ - contractive mapping with $L=\frac{22}{8}$ and the condition (3) of Theorem 3.2 holds trivially. Therefore $f$ satisfies all the hypotheses of Theorem 3.2 and $x=1$ is a fixed point of $f$.

Here we observe that when $L=0$, the inequality (2.4) fails to hold for $x=1$ and $y=2$, we have

$$
\begin{aligned}
\psi\left(s^{3} d(f x, f y)\right) & =\psi\left(\left(\frac{3}{2}\right)^{3} d(f 1, f 2)\right)=\psi\left(\left(\frac{3}{2}\right)^{3}(2)\right)=\psi\left(\frac{27}{8}(2)\right) \\
& \not \leq \psi(2)-\varphi(2)=\psi\left(M_{s}(x, y)\right)-\varphi\left(M_{s}(x, y)\right)
\end{aligned}
$$

for any $\psi$ and $\varphi$, so that $f$ is not an $(\alpha, \beta)-(\psi, \varphi)$-contractive mapping.

Further, we observe that the metric $d$ defined in this example is not a metric in the usual sense, for $x=\frac{1}{3}, y=\frac{1}{5}$ and $z=\frac{1}{2}$ then $d(x, y)=6 \not \leq 2+2=d(x, z)+d(z, y)$.

Example 4.3. Let $X=[0.5, \infty)$ and let $d: X \times X \rightarrow[0, \infty)$ be defined by

$$
d(x, y)= \begin{cases}0 & \text { if } \quad x=y \\ \frac{1}{x}+\frac{1}{y} & \text { if } \quad x, y \in[0.5,1] \\ 4+\frac{1}{x}+\frac{1}{y} & \text { if } x, y \in(1, \infty) \\ 2 & \text { otherwise }\end{cases}
$$

Clearly, $d$ is a $b$-metric space with the coefficient $s \geq \frac{3}{2}$.

Let $A=[0.5,1]$ and $B=[1, \infty)$, we define $f: A \cup B \rightarrow A \cup B$ by

$$
f(x)= \begin{cases}\frac{1}{x} & \text { if } \quad x \in A \\ \frac{1}{2}+\frac{1}{2 x} & \text { if } \quad x \in B .\end{cases}
$$

Now, we have for $x \in A, f x=\frac{1}{x} \in[1, \infty)=B$ which implies that $f A \subset B$ and also for $x \in B, f x=\frac{1}{2}+\frac{1}{2 x} \in[0.5,1]=A$ which implies that $f B \subset A$. Hence $f$ is cyclic. 
We choose $\psi(t)=t, \varphi(t)=\frac{t}{2} \quad t \geq 0$.

For $x \in A$ and $y \in B$ we have

$$
\begin{aligned}
M_{s}(x, y) & =\max \left\{d(x, y), d(x, f x), d(y, f y), \frac{d(x, f y)+d(y, f x)}{2 s}\right\} \\
& =\max \left\{2,2,2, \frac{\frac{1}{x}+\frac{1}{\frac{1}{2}+\frac{1}{2 x}}+4+\frac{1}{y}}{2\left(\frac{3}{2}\right)}\right\} \geq 2,
\end{aligned}
$$

$M_{s}^{\prime}(x, y)=\max \{d(x, y), d(y, f y)\}=\max \{2,2\}=2$,

and $N(x, y)=\min \left\{d(x, f x), d(y, f x\}=\max \left\{2,4+\frac{1}{y}+x\right\}=2\right.$.

Now, we have

$$
\begin{aligned}
\psi\left(s^{3} d(f x, f y)\right) & =\psi\left(\frac{3}{2} d\left(\frac{1}{x}, \frac{1}{2}+\frac{1}{2 x}\right)\right. \\
& =\psi\left(\left(\frac{3}{2}\right)^{3}(2)\right. \\
& \leq 2-\frac{2}{2}+6(2) \\
& \leq \psi\left(M_{s}(x, y)\right)-\varphi\left(M_{s}(x, y)\right)+L N(x, y) .
\end{aligned}
$$

Therefore $f$ is an almost generalized $(A, B)-(\psi, \varphi)$-contractive mapping with $L=6$.

Hence $f$ satisfies all the hypotheses of Theorem 3.4 and $x=1$ is a unique fixed point of $f$.

Further, we observe the following:

if we define

$$
\alpha(x)=\left\{\begin{array}{ll}
1 & \text { if } x \in A \\
0 & \text { otherwise }
\end{array} \quad \beta(x)= \begin{cases}1 & \text { if } x \in B \\
0 & \text { otherwise }\end{cases}\right.
$$

then $f$ is a cyclic $(\alpha, \beta)$-admissible mapping and by (4.2), we have $f$ is an almost generalized $(\alpha, \beta)-(\psi, \varphi)$-contractive mapping. Hence $f$ satisfies all the hypotheses of Theorem 3.1 , along with the hypothesis of Theorem 3.3, and $f$ has a unique fixed point 1.

But for $x=\frac{67}{100}$ and $y=1$, we have

$$
\begin{aligned}
\psi(d(f x, f y)) & =\psi\left(d\left(f \frac{67}{100}, f 1\right)\right)=\psi\left(d\left(\left|\frac{100}{67}-1\right|\right)=\psi\left(\frac{33}{67}\right)\right. \\
& \not \leq \psi\left(\frac{33}{100}\right)-\varphi\left(\frac{33}{100}\right)=\psi(d(x, y))-\varphi(d(x, y)),
\end{aligned}
$$

for any $\psi, \varphi \in \Psi$ so that the inequality (2.1) fails to hold. Hence Theorem 2.1 is not applicable.

\section{R E F E R E N C E S}

1. Ya. I. and Alber m S. Guerre-Delabriere, Principle of weakly contractive maps in Hilbert spaces, New Results in Operational Theory and Its Application, Oper. Theory Adv. Appl., 98, Birkhauser, Basel, (1997), 7-22.

2. S. Alizadeh, F. Moradlou and P. Salimi, Some fixed point results for $(\alpha, \beta)-(\psi, \phi)$-contractive mappings, Filomat., 28(3) (2014), 635-647. 
3. G. V. R. Babu, M. L. SAndhya and M. V. R Kameswari : A note on a fixed point theorem of Berinde on weak contractions, Carpathian J. Math., 24 (2008), 8-12.

4. V. Berinde, Approximating fixed points of weak contractions using the Picard iteration, Nonlinear Anal. Forum, 9 (2004), 43-53.

5. V. BERINDE, General contractive fixed point theorem for ciric- type almost contractions in metric space, Carpathian J. Math., 24 (2008), 10-19.

6. M. Boriceanu, M. Bota and A. Petrusel, Mutivalued fractals in $b$-metric spaces, Cent. Eur. J. Math, 8 (2010), 367 - 377.

7. S. CzerwiK, Contraction mappings in $b$-metric spaces, Acta Math. Inform. Univ. Ostraviensis, 1 (1993), 5 - 11.

8. P. N. Dutta and B. S. Choudhury, A generalization of contraction principle in metric spaces, Fixed Point Theory and Appl., (2008), Article ID 406368, 8 pages.

9. N. Hussain, D. Doric, Z. Kadelburg and S. Radenovic, Suzuki-type fixed point results in metric type spaces. Fixed Point Theory Appl. 2012, 126 (2012). doi:10.1186/1687-1812-2012-126.

10. M. S. Khan, M. Swaleh and S. Sessa, Fixed point theorems by altering distances between the points, Bull. Aust. Math. Soc., 30 (1984), 1-9.

11. W. A. Kirk, P. S. Srinivasan and P. Veeramani, Fixed points for mappings satisfying cyclical contractive conditions, Fixed Point Theory, 4 (2003), 79 - 89.

12. B. E. RhoAdes, Some theorems on weakly contractive maps. Nonlinear Anal. 47 (2001), 2683-2693.

13. J. R. Roshan, V. Parvaneh and Z. Kadelburg, Common fixed point theorems for weakly isotone increasing mappings in ordered $b$-metric spaces, Jour. Nonlinear Sci. and Appl., 7(2014), 229-245.

14. O. Yamaoda and W. Sintunavarat, Fixed point theorems for $(\alpha, \beta)-(\psi, \varphi)$ contractive mappings in b-metric spaces with some numerical results and applications, J.

Nonlinear Sci. Appl. 9 (2016), 22- 33.

G. V. R. Babu

Department of Mathematics

Andhra University

Visakhapatnam-530 003, India.

gvr_babu@hotmail.com

T. M. Dula

Present address :

Department of Mathematics

Andhra University,

Visakhapatnam-530 003, India.

Permanent address :

Department of Mathematics

Wollega University,

Nekemte-395, Ethiopia.

dulamosissa@gmail.com 